\title{
A new method for obtaining bankable and expandable adult-like microglia in mice
}

\author{
Min-Jung You ${ }^{1}$, Chan Rim ${ }^{1}$, Youn-Jung Kang ${ }^{2}$ and Min-Soo Kwon ${ }^{1 *}$ (i)
}

\begin{abstract}
Background: The emerging role of microglia in neurological disorders requires a novel method for obtaining massive amounts of adult microglia. We aim to develop a new method for obtaining bankable and expandable adult-like microglia in mice.

Methods: The head neuroepithelial layer (NEL) that composed of microglial progenitor and neuroepithelial cells at mouse E13.5 was dissected and then cultured or banked. Microglia (MG) isolated from the cultured NEL by magneticactivated cell sorting system were obtained and named NEL-MG.

Results: The NEL included microglia progenitors that proliferate and ramify over time with neuroepithelial cells as feeder. In functional analysis, NEL-MG exhibited microglial functions, such as phagocytosis (microbeads, amyloid $\beta$, synaptosome), migration, and inflammatory response following lipopolysaccharide (LPS) stimulation. NEL was passage cultured and the NEL-MG exhibited a higher expression of microglia signature genes than the neonatal microglia, a widely used in vitro surrogate. Banking or long-term passage culture of NEL did not affect NEL-MG characteristics. Transcriptome analysis revealed that NEL-MG exhibited better conservation of microglia signature genes with a closer fidelity to freshly isolated adult microglia than neonatal microglia. NEL-MG could be re-expandable when they were plated again on neuroepithelial cells.
\end{abstract}

Conclusions: This new method effectively contributes to obtaining sufficient matured form of microglia (adult-like microglia), even when only a small number of experimental animals are available, leading to a broad application in the field of neuroscience.

Keywords: Microglia, Neuroepithelial layer, Subculture, Banking

\section{Introduction}

Since their discovery in the twentieth century by RioHortega, microglia were considered professional phagocytes, similar to macrophages for a long time. However, recent studies propose microglia as a promising target beyond phagocytes under neuroinflammatory/neurodegenerative conditions [1]. Microglia are capable

\footnotetext{
*Correspondence: minsoo100@cha.ac.kr

1 Department of Pharmacology, Research Institute for Basic Medical Science, School of Medicine, CHA University, CHA BIO COMPLEX, 335 Pangyo, Bundang-gu, Seongnam-si, Gyeonggi-do 13488, Republic of Korea

Full list of author information is available at the end of the article
}

of morphological remodeling without any indication of insult or neurodegeneration [2], suggesting a broad functional repertoire, including the maintenance of biochemical homeostasis, neuronal circuit maturation during development, and experience-dependent remodeling of neuronal circuits in the adult brain [3]. The dynamic microglial function lasts during our entire lifetime, and its disturbance can induce abnormal neurodevelopment and neurodegeneration [4].

Despite the increase in knowledge regarding microglial biology [5], lack of a well-validated in vitro culture method remains a bottleneck for establishing a therapeutic strategy targeting microglia. The current in vitro culture of microglia still requires a microglial original author(s) and the source, provide a link to the Creative Commons licence, and indicate if changes were made. The images or other third party material in this article are included in the article's Creative Commons licence, unless indicated otherwise in a credit line to the material. If material is not included in the article's Creative Commons licence and your intended use is not permitted by statutory regulation or exceeds the permitted use, you will need to obtain permission directly from the copyright holder. To view a copy of this licence, visit http://creativecommons.org/licenses/by/4.0/. The Creative Commons Public Domain Dedication waiver (http://creativeco mmons.org/publicdomain/zero/1.0/) applies to the data made available in this article, unless otherwise stated in a credit line to the data. 
cell line and primary culture using neonatal cortex or the adult brain. Microglial cell lines, such as BV2, N9, SIM-A9, Mocha, and MHC3, have an advantage in proliferation and passage culture; however, they differ from adult microglia in genetic and functional aspects due to immortalization [6]. Neonatal microglia have not been artificially manipulated, but have disadvantages regarding their proliferative capacity, and passage culture and banking abilities. Both the microglial cell line and neonatal microglia rarely express key adult microglia genes; in particular, TMEM119 immunoreactivity (IR) is missed $[7,8]$ because microglial cells develop according to a stepwise program [4]. Acute isolation of adult microglia is challenging due to their restricted proliferative capacity, cell viability, and requirement of a greater number of animals [6, 9]. Granulocyte macrophage colony stimulating factor (GM-CSF) and microglia growth supplement (ScienCell) might allow postmortem adult human microglia to proliferate in culture to high passage without several phenotypes alteration [10], but it requires further validation on the microglial signature genes. The iPSC-derived microglia-like cells might also be an alternative method; however, this method is expensive, time-consuming, and not easily accessible [11]. In addition, iPSC-derived microglia-like cells do not originate in the yolk sac and do not show adequate TMEM119 IR compared to adult microglia [12]. Thus, while the demand for microglia for use in research is increasing, there are no current in vitro methodologies that overcome the above-mentioned limitations.

Mouse microglia progenitors begin to migrate from the yolk sac into the CNS at E8.5 and surround the neuroepithelial layer (NEL) in the part corresponding to the head $[1,13]$. Subsequently, mouse microglia progenitors move into the CNS parenchyma until approximately E18.5 and are matured in the CNS microenvironment [14]. As the CNS matures, microglia acquire a ramified morphology, surveying the surrounding parenchyma via movement of dynamic processes [2].

In the support of the idea that microglia progenitor derived from yolk sac should pass NEL to enter CNS parenchyma, we could obtain and co-culture microglial progenitors and neuroepithelial cells together by dissecting mouse E13.5 head NEL, expecting that neuroepithelial cells play a role as feeder cells. CD11bpositive cells were isolated from cultured NEL by magnetic-activated cell sorting (MACS) system and they were named NEL-MG. By this new methodology, we could establish in vitro approach to obtain bankable and expandable matured form of microglia (adult-like microglia).

\section{Materials and methods}

\section{Ethics approval and consent to participate}

All experimental procedures were approved by the Institutional Animal Care and Use Committee (IACUC) of the CHA University (IACUC200116).

\section{Mouse embryonic neuroepithelial layer dissection, culture, and banking}

Uteri from pregnant mice (female C57BL/6, Orient Bio Inc. Seoul, Korea or female B6-EGFP mice, Jackson lab) were dissected and soaked in Hanks' Balanced Salt Solution (HBSS, Invitrogen, USA). The umbilical cord and yolk sac in the mesometrial surface of the uterus were removed using microsurgical instruments under a microscope and embryos were taken out of the uterus. Then, the NEL was dissected carefully using a pair of microsurgical scissors. The shredded tissue was incubated with $1 \mathrm{~mL}$ of $1 \mathrm{X}$ Trypsin-EDTA (ThermoFisher, USA) for $3 \mathrm{~min}$ at $37{ }^{\circ} \mathrm{C}$ in a water bath. After centrifuging at $300 \times g$ for $5 \mathrm{~min}$, the cells were plated onto $25 \mathrm{~cm}^{2}$ flasks coated with poly-Dlysine (Sigma, MO, USA). The cells were cultured in DMEM high glucose medium containing 10\% FBS, $1 \mathrm{X}$ penicillin:streptomycin and $0.1 \%$ GlutaMAX at $37{ }^{\circ} \mathrm{C}$ in a $5 \% \mathrm{CO}_{2}$ incubator. The culture medium was replaced with $5 \mathrm{~mL}$ of fresh growth medium after $24 \mathrm{~h}$. Subsequently, half the culture medium volume was replaced with an equal volume of fresh growth medium twice per week. For banking, NEL were frozen and stored for about ten months in freezing media composed of DMEM with 20\% FBS and 10\% DMSO in liquid nitrogen (about $-196{ }^{\circ} \mathrm{C}$ ). For thawing, the stock vials were dissolved at $37^{\circ} \mathrm{C}$ water bath for $3 \mathrm{~min}$ and diluted with culture medium.

\section{Isolation of microglial cells from NEL}

On $\sim$ day 21 , when stratification was reached, NEL culture flasks were incubated with $1 \mathrm{X}$ trypsin-EDTA for $1 \mathrm{~min}$, resulting in the detachment of an intact layer of cells in pieces. The pellet was resuspended in cold MACS buffer (containing a 1-volume dilution of PBS, 2 mM EDTA, and 0.5\% BSA, pH 7.2), and myelin removal beads (Myelin Removal Beads II, 130-96-733, Miltenyi Biotec) were used according to the manufacturer's protocol. Briefly, cells were incubated with the microbeads coupled anti-CD11b mAb at $4{ }^{\circ} \mathrm{C}$ for $15 \mathrm{~min}$, and then the cells were washed and resuspended in $500 \mu \mathrm{L}$ PB buffer. The magnetically labeled cells were separated by MS column on a magnetic separator. The column was washed thrice with PBS buffer, and the separated cells (NEL-MG) were obtained via positive selection. NEL-MG were resuspended in 
microglial complete culture medium (DMEM, 10\% FBS, $0.1 \%$ GlutaMAX, $5 \mu \mathrm{g} / \mathrm{mL}$ insulin, and $1 \%$ penicillin/streptomycin), transferred to poly-D-lysine-coated plates at a density of $2.5 \times 10^{5}$ cells $/ \mathrm{mL}$, and cultured for subsequent molecular studies.

\section{Adult microglial cell isolation}

Adult microglial cells were isolated from 8 to 10 weekold mice (male C57BL/6, Orient Bio Inc. Seoul, Korea). Enzymatic cell dissociation was performed using an Adult Brain Dissociation Kit (130-107-677, Miltenyi Biotec) according to the manufacturer's instructions. Brain tissue pieces (up to $500 \mathrm{mg}$ ) were transferred into a gentleMACS C tube (130-096-334, Miltenyi Biotec) containing $1950 \mu \mathrm{L}$ of enzyme mix 1 (enzyme $\mathrm{P}$ and buffer $\mathrm{Z}$ ), and then $30 \mu \mathrm{L}$ of enzyme mix 2 (enzyme $\mathrm{A}$ and buffer $\mathrm{Y})$ was added. The gentleMACS $C$ tube was tightly closed and attached upside down onto the sleeve of the gentleMACS Octo Dissociator with Heaters (130-096-427, Miltenyi Biotec), and the appropriate gentleMACS program (37C_ABDK_01) was run. After brief centrifugation to collect the samples at the bottom of the tube, the samples were filtered through a $70 \mu \mathrm{m}$ strainer (130-098462, Miltenyi Biotec), washed with D-PBS, and centrifuged again. The pellet was resuspended in cold D-PBS. The myelin and cell debris were removed using debris removal solution, followed by subsequent removal of erythrocytes using a red blood cell removal solution. Pure adult microglial cells were magnetically isolated using microbeads-coupled anti-CD11b mAb, as stated previously.

\section{Neonatal cortical microglia culture}

Postnatal 1- to 2-day-old B6 mice (Orient Bio Inc. Seoul, Korea) were killed and soaked in $75 \%$ ethanol for $1 \mathrm{~min}$. The cerebral hemispheres were dissected following standard techniques and anatomical landmarks, and the meninges were peeled off. The hippocampus, basal ganglion, and olfactory bulb were carefully removed using microsurgical instruments under a microscope, and the remaining cortical tissue was minced using a pair of microsurgical scissors. The shredded tissue was then incubated with $3 \mathrm{~mL}$ of HBSS (Invitrogen) for $5 \mathrm{~min}$ at $37^{\circ} \mathrm{C}$ in a water bath. After centrifuging at $300 \times g$ for $5 \mathrm{~min}$, the cells were plated onto $25 \mathrm{~cm}^{2}$ flasks coated with poly-D-lysine (Sigma). Mixed glial cells were cultured in DMEM high glucose medium containing $10 \% \mathrm{FBS}$ and $0.1 \%$ GlutaMAX at $37{ }^{\circ} \mathrm{C}$ and $5 \% \mathrm{CO}_{2}$ in an incubator. The culture medium was replaced with $5 \mathrm{~mL}$ of fresh growth medium after $24 \mathrm{~h}$. Subsequently, half of the culture medium volume was replaced with an equal volume of fresh growth medium twice a week. Stratification was reached at the end of this period, and the microglial cells in the upper layer were harvested. On day 14, flasks were incubated with $1 \mathrm{X}$ trypsin-EDTA for $1 \mathrm{~min}$, resulting in the detachment of an intact layer of cells in one piece. The pellet was resuspended in cold MACS buffer (containing 1-volume dilution of PBS, $2 \mathrm{mM}$ EDTA, and $0.5 \%$ BSA, pH 7.2), and then myelin removal beads (Myelin Removal Beads II, 130-96-733, Miltenyi Biotec) were used according to the manufacturer's protocol. Neonatal microglia were isolated with the microbeads coupled anti-CD11b mAb b MACS system. The isolated microglia were resuspended in microglial complete culture medium (DMEM, 10\% FBS, 0.1\% GlutaMAX, $5 \mu \mathrm{g} / \mathrm{mL}$ insulin, and $1 \%$ penicillin/streptomycin) and transferred to poly-D-lysine-coated plates at a density of $2.5 \times 10^{5}$ cells $/ \mathrm{mL}$ for molecular studies.

\section{BV2 cell line and SIM-A9 culture}

Murine BV-2 microglial cells and SIM-A9 (Kerafast, Boston, USA) were maintained and cultured referring to our previous study [15]. The BV2 cells were passaged in an uncoated $100 \mathrm{~mm}$ cell culture dish with DMEM/F-12 (Gibco, cat. \#11320-033) containing 10\% heat-inactivated FBS (Gibco, cat. \#16000-044), 5\% heat-inactivated horse serum (Invitrogen cat. \#16050-122), and 1\% penicillin/ streptomycin (Gibco, cat. \# 15140122). SIM-A9 cells were passaged in an uncoated $100 \mathrm{~mm}$ cell culture dish with DEME/F-12 (Gibco, cat. \#11330-032) containing $10 \%$ heat-inactivated FBS (Gibco, cat, \#16000-044), 5\% heat-inactivated horse serum (Gibco, cat, \# 16050-122), and $1 \%$ penicillin/streptomycin (Gibco, cat, \#15140122). Cells were cultured at $37^{\circ} \mathrm{C}$ in an incubator with $5 \% \mathrm{CO}_{2}$.

\section{Flow cytometry}

The cells were collected and labeled using fluorochromeconjugated monoclonal antibodies recognizing antigens (CD11b-PE, BD Biosciences \#553311) at $4{ }^{\circ} \mathrm{C}$ for $15 \mathrm{~min}$. After labeling, the cells were washed twice in PBS and resuspended in a final volume of $400 \mu \mathrm{L}$. Flow cytometry was performed using a CytoFLEX (Beckman Coulter, cytoflex B4-R1-Vo) and the data were analyzed using CytExpert software.

\section{Quantitative polymerase chain reaction (qPCR)}

Total RNA was extracted using TRIzol reagent (Invitrogen), and evaluated using a NanoDrop 2000 spectrophotometer (Thermo Scientific, ND-2000). cDNA was synthesized using RevertAid First Strand cDNA Synthesis Kit (Thermo, MA, USA). To assess the 
microglia signature in NEL-MG, we analyzed the expression of Tmem119 (Qiagen, Germany, PPM28876A), P2ry12 (PPM04913C), Mertk (PPM34425A), Tgfb1 (PPM02991B), Mafb (PPM05266A), Gpr34 (PPM04860A), Pros1 (PPM31106A), C1qa (PPM24525E), Gas6 (PPM05523A), Csf1r (PPM03625F), Hexb (PPM27125A), and Gapdh (PPM02946E) genes. Other primer information (Trem2, iNOS, TNF- $\alpha, I L-1 \beta, I L-6$, and $C C L 3$ ) is indicated in Additional file 1: Table S2.

\section{Immunocytochemistry}

Cells were seeded on glass cover slips in 24-well plates. Cells were washed with PBS and cultured; the cultured cells were then fixed in $4 \%$ formaldehyde and permeabilized with $0.1 \%$ Triton X-100 for 5-10 min. Indirect immunofluorescence was performed using the following primary antibodies: rabbit anti-PU1 (1:200, Abcam, MA, USA; Ab88082), mouse anti-Ki67 (1:500, BD Pharmingen; 550609), mouse anti-F4/10 (1:200, Abcam; Ab6640), rabbit anti-CX3CR1 antibody (1:200, Abcam), rabbit anti-TMEM119 antibody (1:200, Abcam), rabbit anti-IBA-1 antibody (1:500, Wako, MA, USA), and goat anti-IBA1 antibody (1:500, Abcam; Ab48004). Cells were incubated with the primary antibodies diluted in $0.5 \%$ Triton X-100 in PBS containing 5\% normal donkey serum at $4{ }^{\circ} \mathrm{C}$ overnight. After rinsing thrice with PBS for 5 min, Alexa 488- or Alexa-594-conjugated secondary antibodies (Abcam) were used for detection. Nuclei were counterstained with 4'6-diamidino-2-phenylindole (DAPI; Sigma). Cells without the addition of primary antibodies served as negative controls. Fluorescent images were taken using a confocal microscope (LSM 700, Carl Zeiss, Jena, Germany).

\section{Purification and labeling of synaptosome}

One hemisphere (male C57BL/6, Orient Bio Inc. Seoul, Korea), excluding the cerebellum, ( 200-400 mg), was homogenized in 10 volumes of Syn-PER Synaptic Protein Extraction Reagent (Thermo Fisher Scientific, Part No. 87785) using a $7 \mathrm{~mL}$ Dounce tissue grinder with 10 upand-down even strokes. The homogenate was centrifuged at $1200 \times g$ for $10 \mathrm{~min}$ to remove the cell debris, and the supernatant was centrifuged at $15,000 \times g$ for $20 \mathrm{~min}$ to obtain the synaptosome pellet. The pellets were gently resuspended in the respective reagent. Synaptosomes were conjugated with an amine-reactive dye (pHrodo Red, SE; Thermo Scientific; \#P36600) in 0.1 M sodium carbonate ( $\mathrm{pH} 9.0)$ at room temperature. After $2 \mathrm{~h}$ of incubation, unbound pHrodo was washed-out via multiple rounds of centrifugation and pHrodo-conjugated synaptosomes were resuspended in isotonic buffer containing 5\% DMSO for subsequent freezing (Invitrogen, \#P36600).

\section{Phagocytosis assay}

To assess the phagocytic activity, NEL-MG at a density of $2 \times 10^{5}$ cells $/ \mathrm{mL}$ were seeded on a $12 \mathrm{~mm}$ coverslip in 24-well cell culture dishes. NEL-MG were treated with $2 \mu \mathrm{L}$ of red fluorescent latex beads $(2 \mu \mathrm{m}$, Sigma-Aldrich, St. Louis, MO, USA), HiLyte ${ }^{\mathrm{TM}}$ Fluor 488-labeled amyloid $\beta$ peptide $25-35$ ( $2 \mu \mathrm{L}$ ), or pHrodoconjugated synaptosomes for $2 \mathrm{~h}$ at $37{ }^{\circ} \mathrm{C}$. HiLyte ${ }^{\mathrm{TM}}$ Fluor 488-labeled amyloid $\beta$ peptide 25-35 (Anaspec, AS-633308) was prepared according to the manufacturer's protocol. Phagocytic activity was then stopped by adding $2 \mathrm{~mL}$ of ice-cold PBS. The cells were washed twice with ice-cold PBS, fixed, stained with a microglial marker (IBA-1), and counterstained with DAPI. The cells were analyzed using confocal microscopy (TCS SP5, Leica) and a DeltaVision fluorescence microscopy system (Applied Precision).

\section{Scratch wound assay}

NEL-MG were seeded onto 24-well plates in a $100 \%$ confluent monolayer until they were $95 \%$ confluent and were wounded by making a perpendicular scratch with a $200 \mu \mathrm{L}$ pipette tip. The cells were replenished with fresh growth medium and wound closure was documented by photographing the same region at different times $(0-6 \mathrm{~h})$. The wound area was calculated as the open wound area/ total area.

\section{Cytokine profiles}

The supernatant was analyzed using a Proteome Profiler Mouse Cytokine Array Panel A Kit (R\&D Systems; catalog number ARY006) at the baseline and after LPS stimuli according to the manufacturer's indications. Images were captured using a LAS $4000\left(\right.$ lmageQuant $\left.^{\mathrm{TM}}\right)$ and analyzed using ImageJ software program.

\section{RNA sequence and data analysis}

RNA quality was assessed with an Agilent 2100 bioanalyzer using an RNA 6000 Nano Chip (Agilent Technologies, Amstelveen, Netherlands). The library construction was performed using a QuantSeq 3'-mRNA-Seq Library Prep Kit (Lexogen, Inc., Austria) according to the manufacturer's instructions. High-throughput sequencing was performed as 75 single-end sequences using NextSeq 500 (Illumina, Inc., USA). QuantSeq 3'-mRNA-Seq reads were aligned using Bowtie2 (Langmead and Salzberg 2012). Differentially expressed genes were determined based on the counts from unique and multiple alignments using coverage in Bedtools (Quinlan AR, 2010). The Read Count data were processed based on the quantile normalization method using Edge $\mathrm{R}$ within 
R (R Development Core Team 2016) using Bioconductor (Gentleman et al. 2004). Gene classification was based on searches done using DAVID (http://david.abcc.ncifcrf. gov/) and Medline databases (http://www.ncbi.nlm.nih. gov/).

\section{Neurospheroid culture}

The whole brain was dissected from postnatal 1- to 2-dayold mice (C57BL/6, Orient Bio Inc. Seoul, Korea). Brain tissues were then cut and chopped in HBSS (Gibco) for $3 \mathrm{~min}$. Next, the dissected brain was centrifuged at $300 \times g$ for $5 \mathrm{~min}$, after which the pellet was resuspended and washed twice in D-PBS. To detect the capacity for selfrenewal, $10^{5}$ cells were plated onto each well of a 25T-flask in growth-promoting medium: DMEM/F12 containing B27 supplement $(\times 50)$, minus vitamin A (12587010, ThermoFisher), $50 \mathrm{ng} / \mathrm{mL}$ FGF2 (100-18B, PEPROTECH), and $50 \mathrm{ng} / \mathrm{mL}$ EGF (AF-100-15, PEPROTECH). Cultures were maintained at $37{ }^{\circ} \mathrm{C}$ in a $5 \% \mathrm{CO}_{2}$ incubator for neurospheroid (NS) formation.

\section{CellTracker labeling}

NEL-MG were labeled using CellTrackerRed CMTPX (Invitrogen) before mixing with NS. Labeling was performed according to the manufacturer's indications.

\section{Mixing of NEL-MG with neurospheroids}

NEL-MG were co-mixed with dissociated NS or posttreated with the already formed NS. NS were mixed with NEL-MG in a 9:1 ratio $\left(1.8 \times 10^{6} \mathrm{NS}: 2 \times 10^{5} \mathrm{NEL}-\mathrm{MG}\right)$ or a 7:3 ratio $\left(1.4 \times 10^{6} \mathrm{NS}: 6 \times 10^{5} \mathrm{NEL}-\mathrm{MG}\right)$ in DMEM/F12 containing B27 supplement $(\times 50)$, $50 \mathrm{ng} / \mathrm{mL}$ FGF2, and $50 \mathrm{ng} / \mathrm{mL}$ EGF. After the addition of NEL-MG, the plates were maintained under static conditions in a shaking incubator $(70 \mathrm{rpm})$ at $37^{\circ} \mathrm{C}$ with $5 \% \mathrm{CO}_{2}$.

\section{Statistical analysis}

The statistical significance of differences between groups was assessed using an unpaired t-test or one-way analysis of variance using GraphPad Prism version 7 for Mac (GraphPad, La Jolla, CA). A post hoc test was performed using one-way analysis of variance when the p-values were significant $(p<0.05)$.

\section{Results}

Expandable and ramified microglial cells are generated from mouse E13.5 head neuroepithelial layer

To determine the optimal period for obtaining a high yield of microglia progenitors, we dissected head NEL from mouse E9.5, E13.5, and E17.5 and cultured them (Fig. 1A). Immunofluorescence analyses revealed that the highest numbers of microglia progenitors were obtained from the mouse E13.5 NEL compared to that of mouse E9.5 or E17.5 (Additional file 1: Fig. S1). The flow cytometry results showed that E13.5 NEL contain more CD11b-positive cells than the brain cortex at E13.5 (Additional file 1: Fig. S1). We next examined whether microglia progenitors were expandable and ramified over time in this culture system. The proportion of CD11b-positive cells increased from 14.2 up to $54.25 \%$ after 21 days of culture (Fig. 1B). The immunofluorescence results showed that the number of microglial cells that were IBA-1-, PU1-, and F4/80-positive increased over time. The percentage of double-positive cells (Ki67 and IBA-1) also increased to 50\%, suggesting that microglia have a high proliferative capacity in this culture system (Fig. 1C).

\section{Functional validation of magnetically isolated CD1 1 b-positive cells (NEL-MG)}

CD11b-positive cells were isolated using a MACS system from the E13.5 NEL (NEL-MG). NEL-MG stained positive for microglia markers with IBA-1 and CX3CR1. NEL-MG showed a relatively weak TMEM119 IR; however, they did not express Ki67, indicating that NEL-MG alone did not proliferate without neuroepithelial cells (Fig. 2A). Next, we conducted microglia-specific functional assays, including phagocytosis, migration, and cytokine/chemokine release using NEL-MG. NEL-MG showed phagocytic function against stimuli, such as synaptosome, amyloid $\beta$, and microbeads (Fig. 2B). In addition, NEL-MG had higher phagocytic activity than BV2, neonatal microglia, and adult microglia (Additional file 1: Fig. S2). The scratch wound assay results showed that NEL-MG could migrate (Fig. 2C). Based on the cytokine and chemokine profiles, unstimulated NEL-MG released

\footnotetext{
(See figure on next page.)

Fig. 1 Expandable microglia generation from the embryo neuroepithelial layer. A Mouse head neuroepithelial layer (NEL) was dissected at E13.5 and was cultured for 21 days (d). B Microglia progenitors proliferated and ramified over time. $\mathbf{C ~} \mid \mathrm{BA}-1^{+} \mathrm{Ki} 67^{+}$cells were stained and the ratio of $\mathrm{IBA}-1^{+} \mathrm{Ki} 67^{+}$cells/IBA-1 ${ }^{+}$cells increased over time. The number of PU1- and F4/80-positive cells also increased with the progression of the culture. TMEM 119 was stained weakly at $21 \mathrm{~d}$. Scale bar $=100 \mu \mathrm{m}$. Proliferative microglia (IBA- $1^{+} \mathrm{Ki} 67^{+}$cells) were counted using microscopy. Sampled areas were selected randomly from $100 \times$ fields in three independent experiments. A post hoc test was conducted using Tukey's multiple comparison tests. ${ }^{* *} p<0.01,{ }^{* * *} p<0.001$ compared to $1 d,{ }^{*} p<0.05$ compared to $7 \mathrm{~d}$
} 

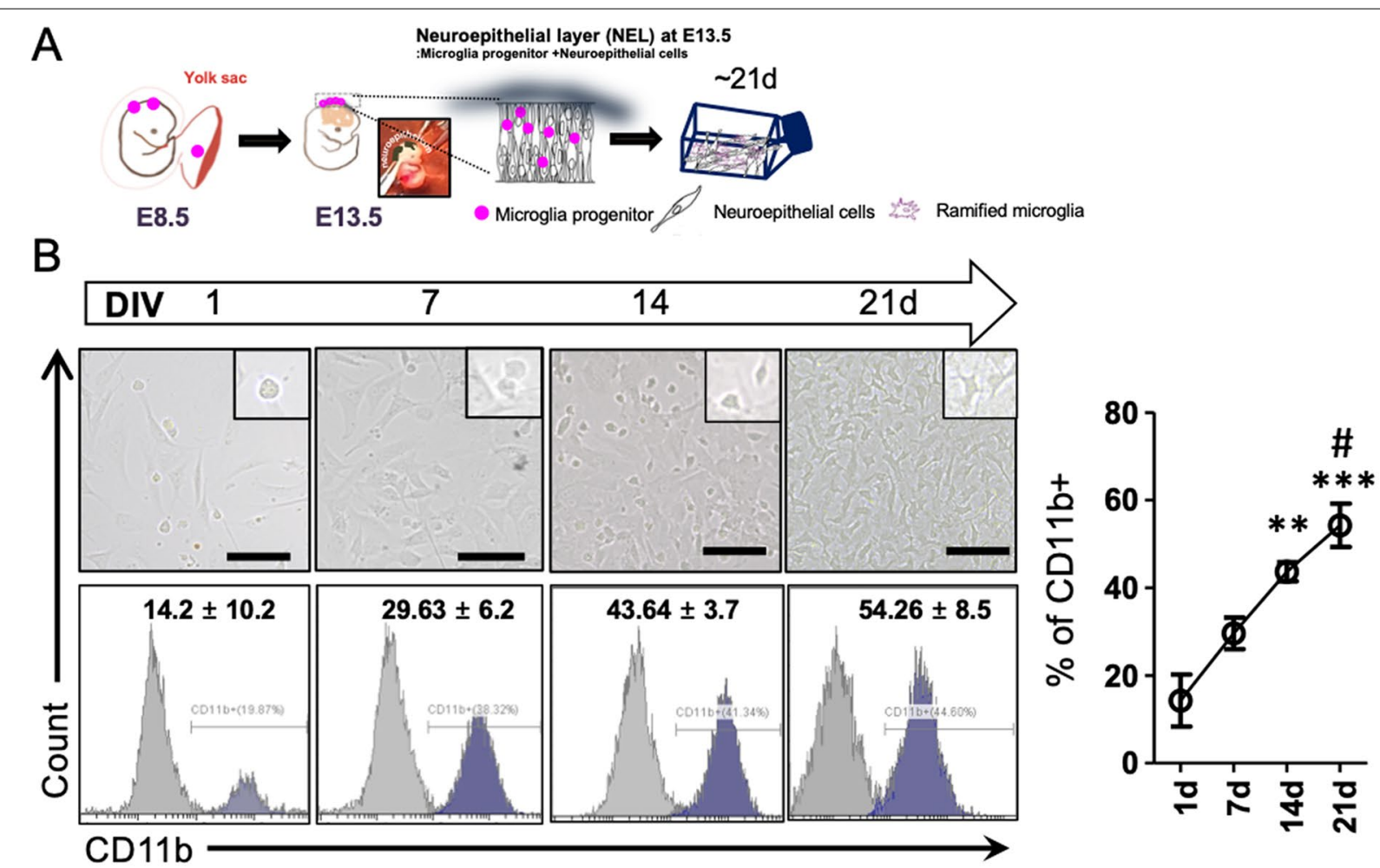

C
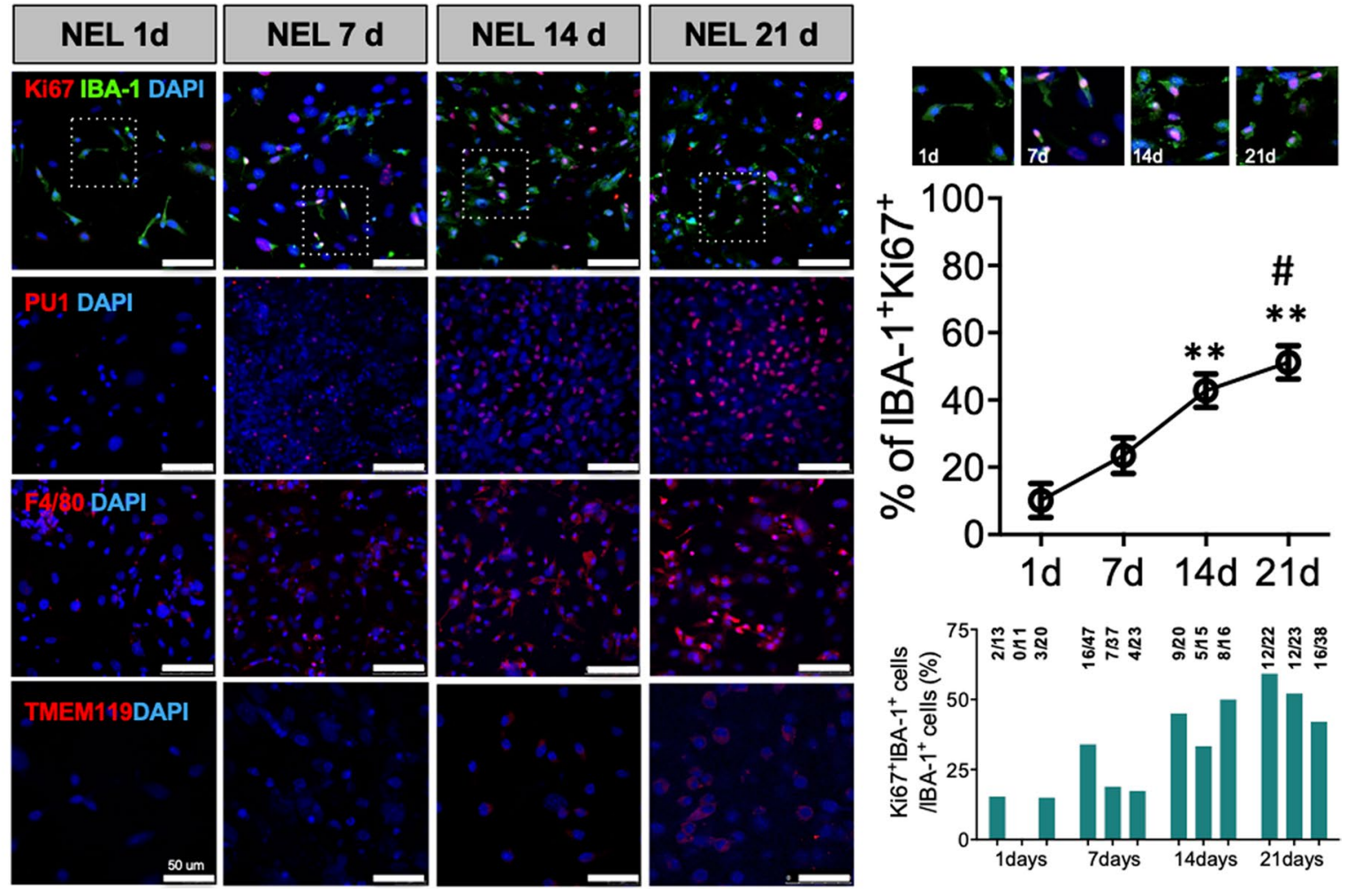

Fig. 1 (See legend on previous page.) 
detectable cytokines and chemokines, including the C-X-C motif chemokine ligand 10 (CXCL10), CXCL1, $\mathrm{C}-\mathrm{C}$ motif chemokine ligand 12 (CCL12), CCL3, TNF$\alpha$, and IL-6. The addition of endotoxin (LPS) triggered the release of chemokines and cytokines above baseline levels (untreated) (Fig. 2D), which was verified at the transcriptional level, with an increase in the mRNA expression of iNOS, TNF- $\alpha$, IL-1 $\beta$, IL- 6 , and CCL3 (Fig. 2E), showing that changes in RNA translated into changes in protein levels. Therefore, NEL-MG acted as a substrate for studying the functional changes in microglia to screen for inflammatory modulators.

Long-term passage culture of NEL and a higher expression of NEL-MG on microglia signature genes

After validating the microglial function of NEL-MG, we analyzed whether the long-term passage culture of E13.5 NEL was possible. Whenever the cells reached stratification, the cells in one $25 \mathrm{~T}$ flask were passage cultured into two $25 \mathrm{~T}$ flasks. As we undertook this passage culture, we obtained approximately double the microglial cells until at least passages (P) 6-7 (first obtained microglial cell number $\times 2^{\mathrm{n}}, n=$ passage number). This was because we could obtain about $50 \%$ of CD11b-positive cells from NEL, despite the long-term passage culture (Fig. 3A and Additional file 1: Fig. S3). However, the required time for the next passage gradually increased as the passageculture progressed and the period without obtaining twice as many cells only occurred after passage 7 . Therefore, the total number of microglia obtained when we used a cutoff of P7 (100 days) was 30 times higher than using the neonatal microglia method (Additional file 1: Fig. S3).

We next examined the alterations in the microglial signature genes in NEL-MG obtained from longterm cultured NEL to determine the optimal period to mimic adult microglia. NEL-MG were obtained by isolation from P0 (21 days), P1 (30 days), P2 (40 days), P3 (50 days), P4 (60 days), P7 (100 days), P8 (120 days), and P10 (180 days) of NEL culturing, and the expression of microglial signature genes in each NEL-MG group was compared to that in neonatal microglia. The expression of most of the microglia identity genes was stably maintained and was higher than in neonatal microglia up to P10 ; however, the expression levels of $T g f b 1$, $M a f b$, Trem2, and Csf1r were significantly lower at P8 than P0 (Fig. 3B). The expression level of the representative adult microglia gene, Tmem119, gradually increased until isolation from the P3 NEL and decreased afterward; however, it remained approximately threefold higher than Tmem119 expression in neonatal microglia. Our data strongly suggest that adult microglia-like NEL-MG were obtainable from cultured NEL for P0-P7 (about 21-100 days). However, the IR of microglial markers, such as IBA-1, CX3CR1, and TMEM119, and their phagocytic function did not change, regardless of the NEL culture duration (Fig. 3C and D).

\section{Banked NEL-MG stably maintain the expression of overall microglia signature genes}

Animals must be sacrified whenever we need to obtain primary microglia; therefore, whether NEL is bankable needs to be determined. NELs $\left(1 \times 10^{7}\right.$ cells $\left./ \mathrm{mL}\right)$ were banked for approximately 10 months in media composed of Dulbecco's modified Eagle medium (DMEM) with $20 \%$ fetal bovine serum (FBS) and 10\% DMSO (banked NEL, bNEL). As shown in Fig. 4A, after thawing, the bNEL proliferated well, similar to fresh NEL. To investigate whether the banking timing affected NEL-MG characteristics, NELs were banked at different passages (P1, P2, P3, and P4) and were cultured for 70 days after thawing. Each NEL-MG group isolated from bNEL at different time points $(7,14,21$, 40 , and 70 days) showed a stable expression of microglia signature genes. The expression levels of these genes remained higher than those in neonatal microglia (Fig. 4B); however, TMEM119 mRNA expression showed a decreasing trend at 70 days (not significant). Csf1r expression was also reduced after thawing; however, its mRNA level was gradually restored as the culture progressed. NEL-MG isolated from bNEL did not show alterations in the phagocytic function compared to NEL-MG isolated from fresh NEL (Fig. 3D and C).

\footnotetext{
(See figure on next page.)

Fig. 2 Functional validation of NEL-MG. A CD11 b+ cells were isolated from cultured NEL for 21 days using an MACS system (NEL-MG) and NEL-MG showed IBA-1, CX3CR1, and TMEM119 IR, but not Ki67 IR. B A phagocytosis assay using microbeads, synaptosome, and amyloid $\beta$ was conducted to assess NEL-MG. C Migration performance was assessed using a wound healing assay. D Cytokines and chemokines released in the supernatant of NEL-MG in response to LPS challenge. E Transcription in NEL-MG as a baseline (CON) and after LPS stimulation (LPS) ( $n=3$ ). The experiment was conducted independently three times. Scale bar $=50 \mu \mathrm{m}$. An unpaired $t$-test was conducted to compare the two groups. ${ }^{* * *} p<0.0001 \mathrm{compared}$ to the control
} 


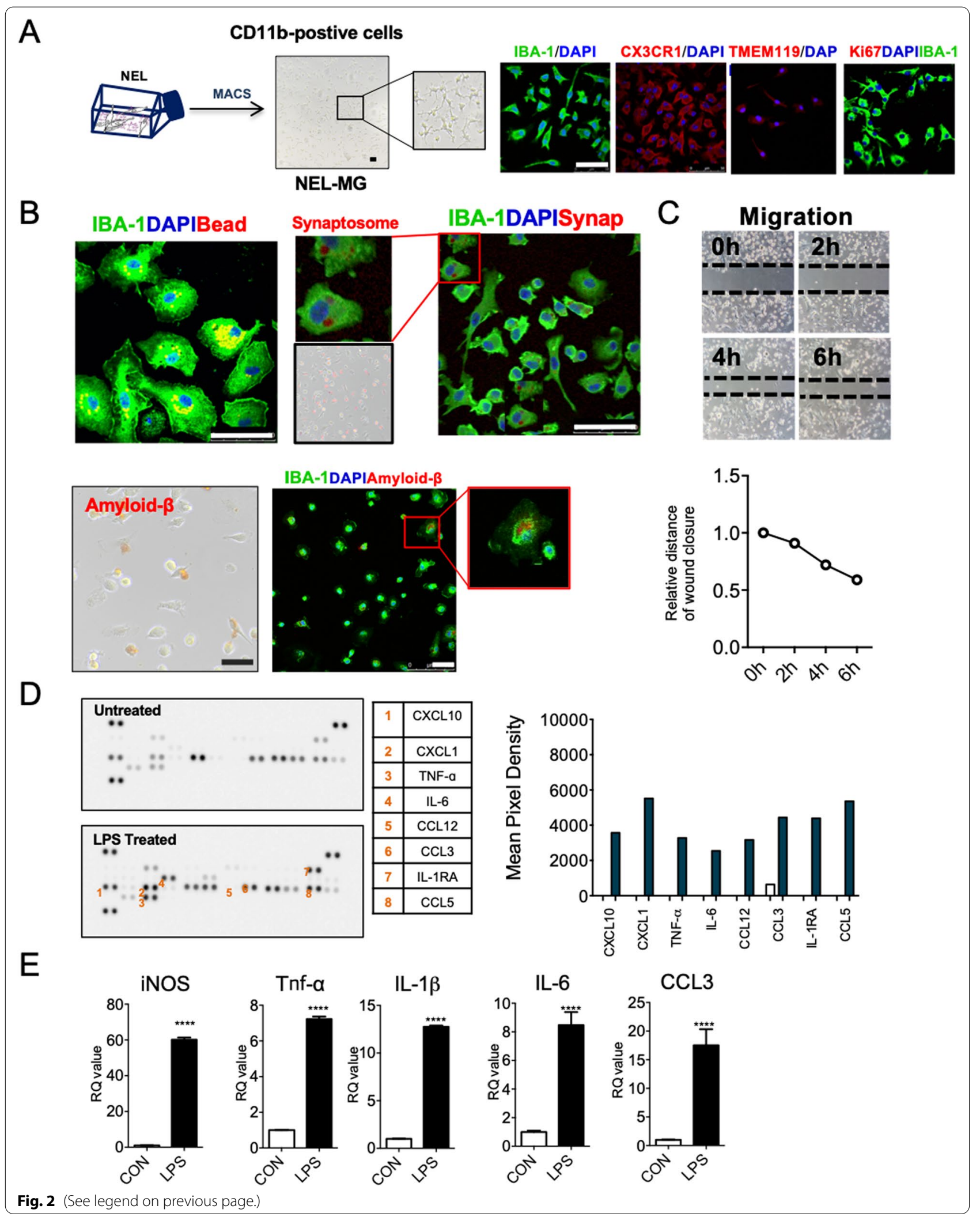


Transcriptome analysis revealed that NEL-MG are closer to adult microglia than neonatal microglia

To more thoroughly characterize NEL-MG compared to the BV2 cell line, neonatal microglia, and adult microglia, we conducted transcriptome analysis. Using $3 \mathrm{D}$ principal component analysis (PCA) of the whole transcriptome, each group was classified into distinct clusters based on their identity. NEL-MG and bNELMG were clustered together and were distinct from the BV2 cell line and neonatal microglia. The component explaining the majority of the dataset variance was PC1, which most prominently distinguished neonatal microglia and NEL-MG from adult microglia and occupied an edge position along PC1. The second principal component (PC2) uniquely distinguished the BV2 cell line from neonatal microglia and adult microglia. The third principal component (PC3) uniquely distinguished NEL-MG from neonatal microglia (Fig. 5A). The PCA Euclidean distances to the adult microglia were calculated between all pairs of points in each group, using $\mathrm{PC} 1, \mathrm{PC} 2$, and $\mathrm{PC} 3$ on the microglia signature genes (Additional file 1: Table S1) [8, 16]. NELMG and bNEL-MG were significantly closer to adult microglia than BV2 and neonatal microglia (Fig. 5B). The hierarchy analysis of the microglia signature genes showed that NEL-MG and bNEL-MG formed a cluster and had more similarities to adult microglia than to BV2 cell line and neonatal microglia (Fig. 5C). The overall expression changes (z-score normalized) in microglia signature genes were compared among the BV2 cell line, neonatal microglia, NEL-MG, and adult microglia (mean \pm standard deviation [SD]). Both NELMG and adult microglia were localized in the opposite area (positive Z-score) from neonatal microglia and the BV2 cell line (negative Z-score). The overall expression of microglia signature genes in the NEL-MG was elevated to the same expression level as that of the genes in adult microglia (Fig. 5D). In a normalized gene expression plot, the scatters in NEL-MG were more overlapped and closer to the diagonal line than neonatal microglia (Additional file 1: Fig. S4).

We next performed differential gene expression analysis (DEG, absolute fold changes $>1.5, \mathrm{p}<0.05$ ) and identified that NEL-MG shared 789 genes (up DEG: 302, down DEG: 487) with adult microglia (Fig. 5E). Gene ontology (GO) analysis revealed the gene subsets of the immune system process, actin filament organization, intracellular signaling transduction, and innate immune response (Fig. 5F). The KEGG pathway of the shared genes indicated Fc gamma R-mediated phagocytosis, leukocyte transendothelial migration, and chemokine signaling pathway (Fig. 5G). NEL-MG showed 2782 DEG (2,057 upregulated and 725 downregulated) compared to neonatal microglia (Fig. $5 \mathrm{H}$ ), and the GO analysis revealed gene subsets, including those involved in endocytosis, cell adhesion, cell migration, cholesterol biosynthetic process, cholesterol metabolic process, and the negative regulation of cell proliferation (Fig. 5I). To better understand the differential expression level of microglia signature genes between NEL-MG and neonatal microglia, we compared the fold change of the expression of microglia signature genes (Additional file 1: Table S1), and 18 genes were significantly changed (Fig. 5J).

\section{Comparison of NEL-MG with adult microglia regarding microglia signature genes}

To further confirm the transcriptome analysis, we compared the mRNA expression of microglial signature genes among the BV2 cell line, SIM-A9 cell line, neonatal microglia, NEL-MG (P3, 50 days), and acutely isolated adult microglia. qRT-PCR analyses demonstrated that NEL-MG had higher or similar expression levels of microglia signature genes, including $T g f b 1, T g f b r 1$, Trem2, Csf1r, C1qa, Pros1, and Gas6 (Fig. 6A). Although Tmem119 and P2ry12 expression levels of NEL-MG did not reach those in adult microglia, they were higher than in neonatal microglia. In addition, the intensity of TMEM119 IR in NEL-MG (P3, 50 days) was similar to that in adult microglia, whereas BV2 and neonatal microglia did not show TMEM119 IR (Fig. 6B).

\section{Cultured NEL-MG showed re-expansive capacity on neuroepithelial cells}

To further explore whether cultured NEL-MG could be proliferative again on neuroepithelial cells as a feeder,

\footnotetext{
(See figure on next page.)

Fig. 3 Stable maintenance and superiority of NEL-MG on adult microglial genes despite the long-term passage culture. When NEL reached stratification, cells in one 25 T Flask were divided into two 25 T flasks, and then cultured for 21 days (d) for flow cytometry. A The ratio of CD11 b+ microglia among NEL was approximately 50\%, showing a stable maintenance ratio, despite the long-term passage culture ( $n=3$, mean \pm SD) in flow cytometry. B NEL-MG were isolated based on the NEL passage culture (P0, P1, P2, P3, P4, P7, P8, and P10) and alterations in the adult microglial genes were examined compared to neonatal microglial cells. The experiment was performed three times independently ( $n=3$ per group) and the data represent mean \pm standard error of the mean (SEM). A post hoc test was conducted using Dunnett's multiple comparison test. ${ }^{*} p<0.05$, ${ }^{* * *} p<0.0001,{ }^{* * * *} p<0.00001$ compared to the $21-\mathrm{d}$ group. Gray zone represents a value $=1$ (neonatal microglia). C IBA-1, CX3CR1, and TMEM119 IRs and phagocytic function were not different in NEL-MG isolated from NEL at P0 (21d), P6 (90 d), and P10 (180 d). Scale bar = 50 $\mu$ m
} 
A
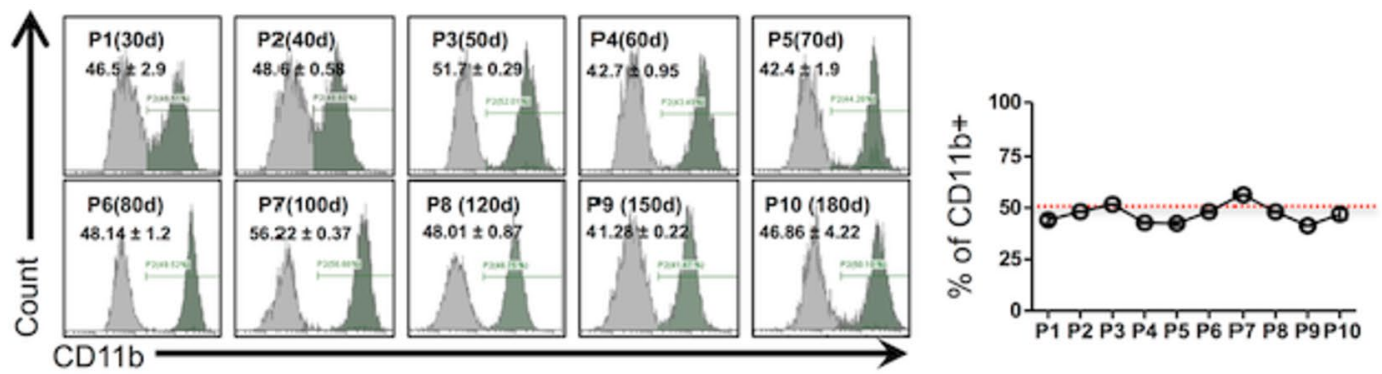

B
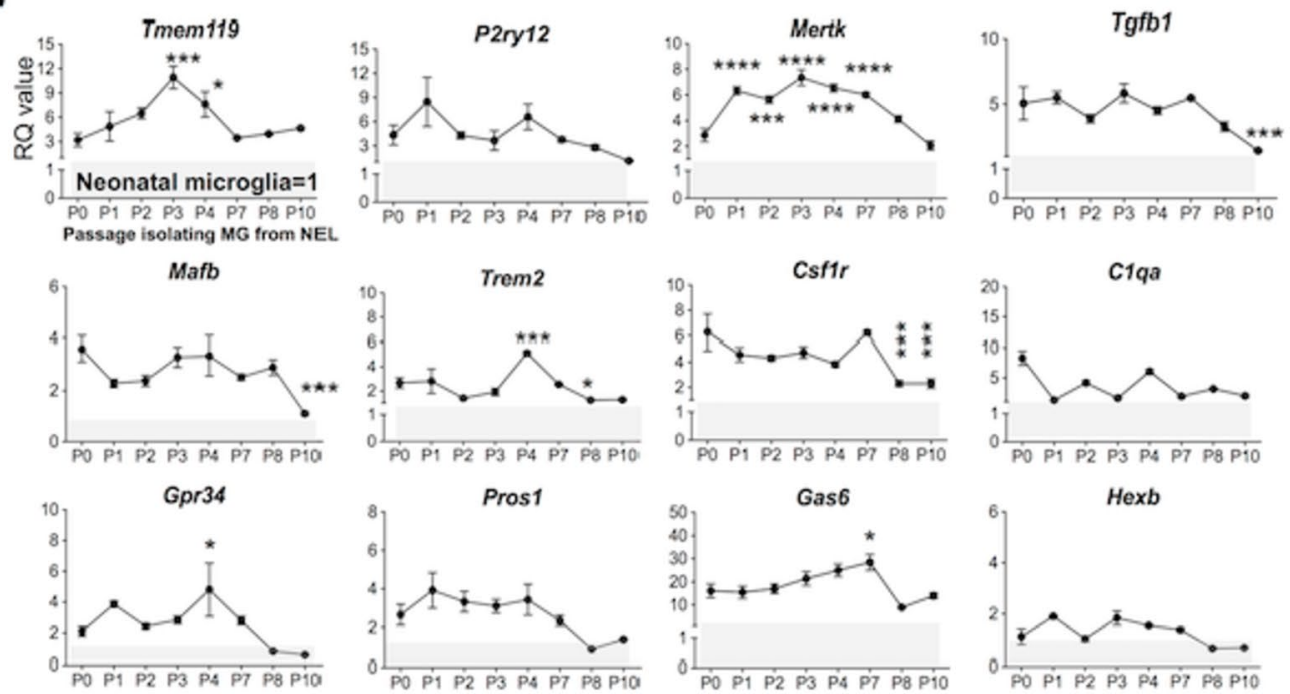

C
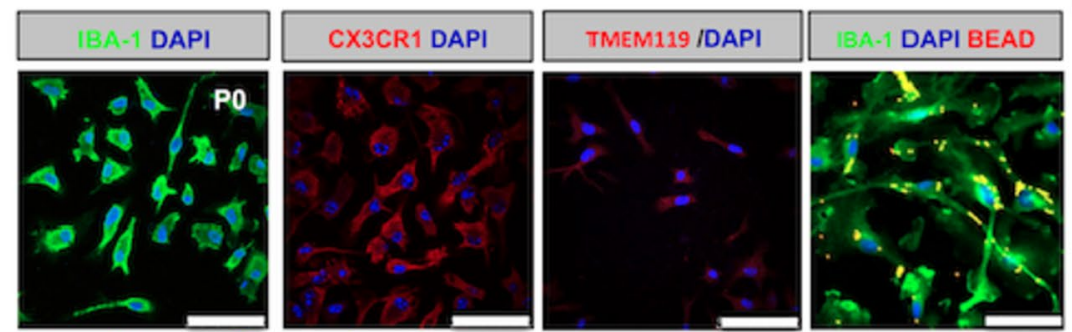

D
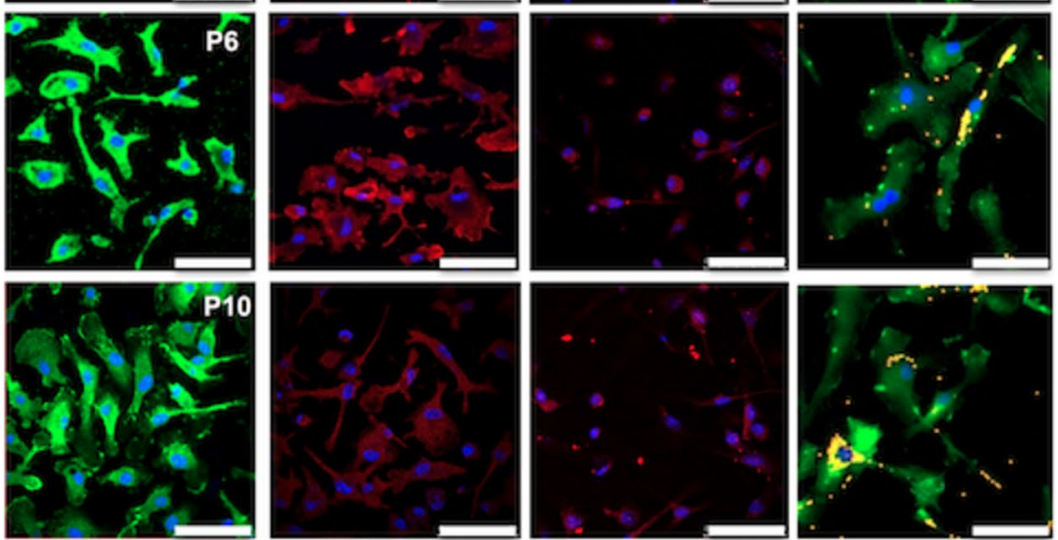

$n=100$

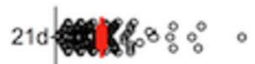

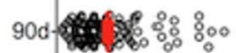

$180 \mathrm{~d}$ (4)
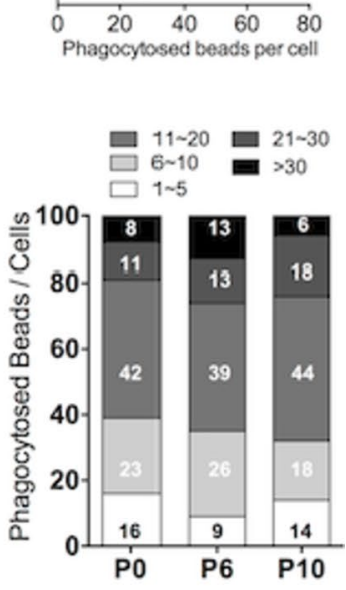

Fig. 3 (See legend on previous page.) 
we isolated and cultured NEL-MG derived from NEL of GFP mice (GFP-expressing NEL-MG). Then, the cultured GFP-expressing NEL-MG were transferred on the remaining neuroepithelial cells (from B6 mice) after NEL-MG isolation. As shown in Fig. 7A and B, using flow cytometry, although the relative ratio of GFP-expressing NEL-MG did not increase significantly over time, we could confirm that $\mathrm{GFP}^{+} \mathrm{Ki} 67^{+}$cells were gradually increased in the immunofluorescence study. Therefore, already cultured NEL-MG could have a proliferative capacity when they were plated again on neuroepithelial cells.

\section{Tracking of NEL-MG within neurospheroids}

Taking advantage of the high cell yield, we established CellTracker-tagging NEL-MG (red) and mixed them with neurospheroids (NS) at different ratios and times. NELMG were mixed evenly with NS when we co-mixed them (Additional file 1: Fig. S5). We could easily detect red color of NEL-MG within NS.

\section{Discussion}

Our robust protocol allowed us to obtain bankable and expandable microglial cells, leading to the production of a high yield of microglial cells that were closer to adult microglia than neonatal microglia based on PCA. We based our study on the developmental process, in which mouse microglial cells were derived from yolk sac macrophage precursors and colonized into nascent parenchyma via the head neuroepithelial layer between E8.5 and E18.5 [1]. The neuroepithelial layer in this area includes mainly two types of cells: neuroepithelial cells and microglia progenitors. Thus, we obtained microglial cells by dissecting and culturing the neuroepithelial layer cells with lower cell heterogeneity than the brain cortex, which is composed of neurons, microglia, astrocytes, and oligodendrocytes, making it possible to obtain purer microglial cells. Neuroepithelial cells were assumed to act as feeders for microglia proliferation and maturation, which was evidenced by our data, showing that microglia progenitors proliferate with Ki67-positive labeling on neuroepithelial cells, although NEL-MG alone failed to proliferate. Therefore, neuroepithelial cells within a co-culture system play a critical role as feeders for microglial proliferation and maturation. This result also indicates that the alone cultured NELMG can have proliferative capacity again as microglia progenitors by the neuroepithelial support. Similarly, an astrocyte feeder layer or an astrocyte-conditioned medium makes microglia morphology and other properties resemble adult microglia more closely $[17,18]$. Compared to the astrocyte feeder support, our protocol has advantages in a simpler methodology (dissection and culture of NEL at E13.5) without independent preparation process of feeder cells. In addition, NEL was bankable and passage culture of NEL was possible, resulting in high yields of microglia obtainment.

Microglia rely on sustained CSF-1 stimuli, which is a well-established trophic cue for survival [19], and TGF- $\beta$ family members promote microglial survival and development [8]. Along with CSF-1 and TGF$\beta$, cholesterol is another main factor that permits the robust survival of highly ramified adult microglia [17]. Our GO results indicate that NEL-MG shared pathways in general microglia function with adult microglia compared to neonatal microglia. However, NEL-MG was distinct from neonatal microglia, especially regarding the cholesterol biosynthetic process and metabolic process. Given the link between an inadequate delivery of cholesterol and generalized microglial dysfunction, the cholesterol metabolic system might be involved in key features in a more matured form of NEL-MG than neonatal microglia. The expression of microglial signature genes, including TMEM119, P2RY12, and $C X 3 C R 1$, is gradually reduced with age or in neurodegenerative diseases, suggesting the loss of homeostatic microglial function [20]. De novo synthesized cholesterol transported via APOE- or APOJ-containing nanodiscs within the CNS $[21,22]$ contribute to amyloid $\beta$ clearance and microglial cholesterol content [23]. Increased lipid metabolism is required to fuel protective cellular functions, such as phagocytosis [24]. Thus, we speculate that alterations in the cholesterol metabolic system might be associated with the characteristics of the matured form of NEL-MG, which is closer to adult microglia.

There are several aspects that require further exploration regarding NEL-MG. First, we need to clarify how neuroepithelial cells make microglia progenitor

\footnotetext{
(See figure on next page.)

Fig. 4 Validation of NEL-MG isolated from banked NEL. A NEL were banked for about 10 months and thawed. The thawed NEL were cultured for 1, 7, 14, 21, 70 days (d) and NEL-MG were isolated at each time point for flow cytometry. B Banked NEL at different passages (P1, P2, P3, and P4) were thawed and cultured for $7,14,21,40$, and $70 \mathrm{~d}$. NEL-MG were isolated at each time point for qPCR ( $n=4$ per group) (black circle: mean value of P1, P2, P3, and P4) and the data represent mean \pm standard error of the mean (SEM), ${ }^{*} p<0.05,{ }^{* *} p<0.001$ compared to the pre-banked group. A post hoc test was conducted using Dunnett's multiple comparison tests. C Immunofluorescence study of IBA-1, CX3CR1, and TMEM119 IR and a phagocytosis assay were conducted using banked NEL-MG (70 d). Scale bar $=50 \mu \mathrm{m}$
} 


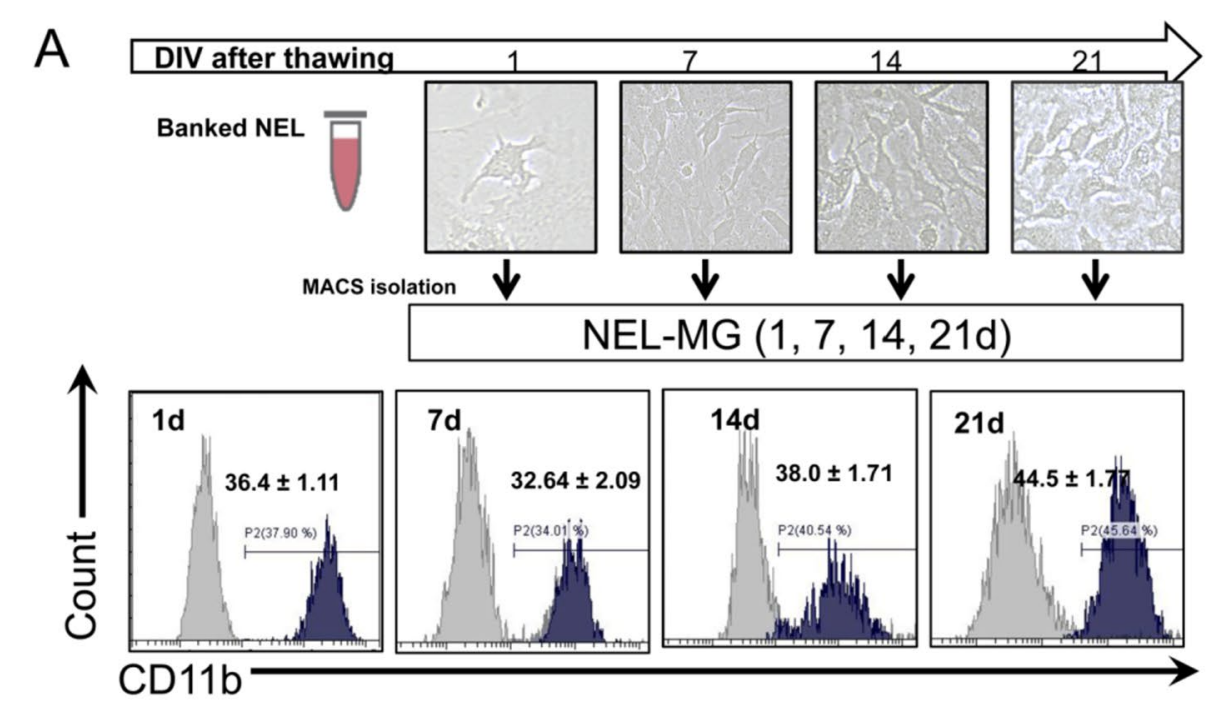

B

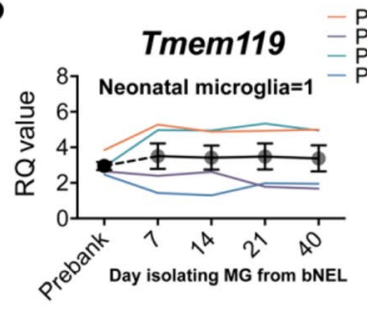

Mafb

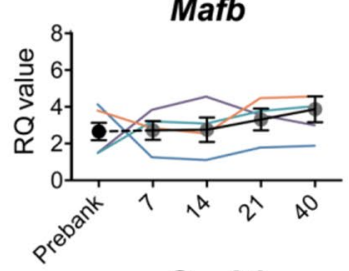

Gpr34

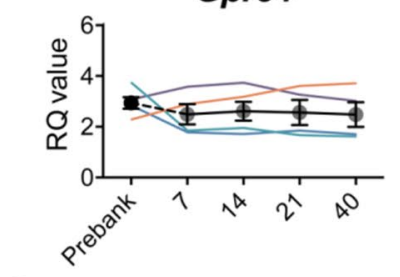

C

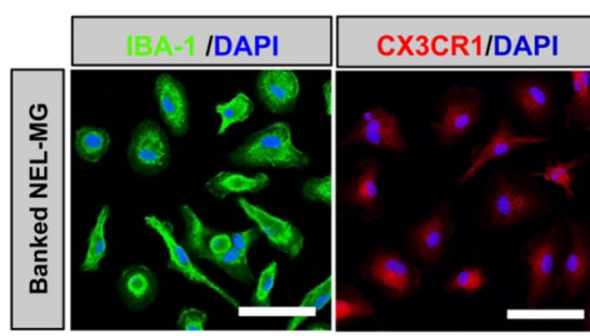

P2ry12

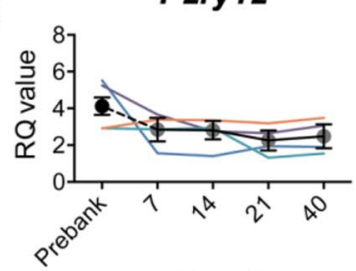

Trem2

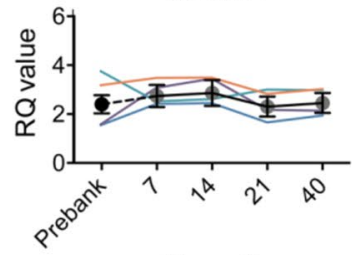

Pros1

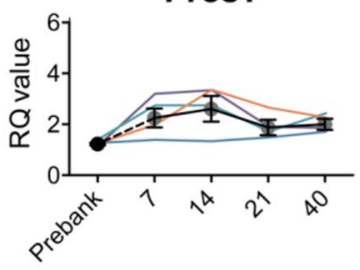

Mertk

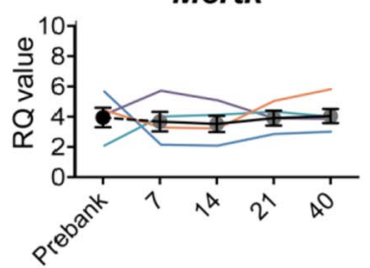

Csf1r

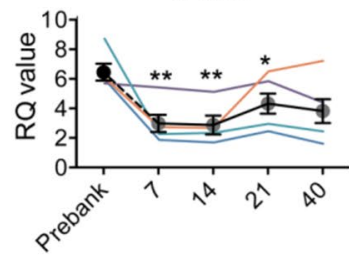

Gas6

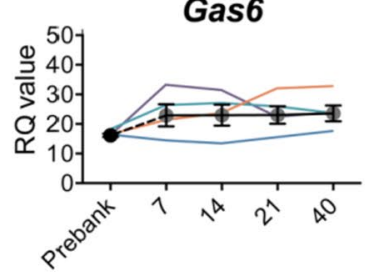

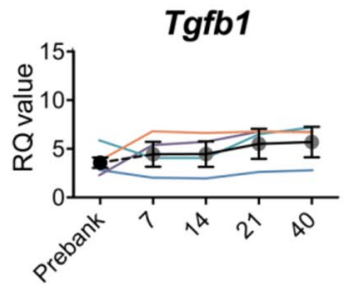

C1qa

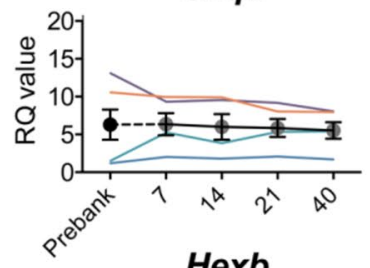

Hexb

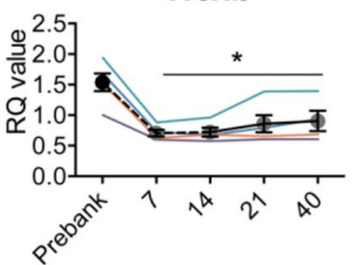

Fig. 4 (See legend on previous page.)
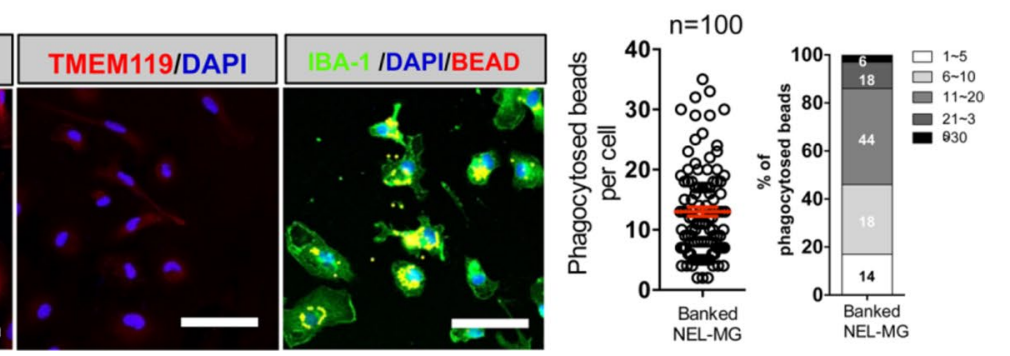
to be mature and proliferate. Like astrocyte-derived factors, neuroepithelial cells might play a role for microglia survival, maturation, and proliferation. Mature microglia require continuous signaling from the healthy CNS [17]. Neuroepithelial cells function as stem cells and can differentiate to astrocyte, oligodendrocytes, and neuronal precursors, suggesting that neuroepithelial cells have a potential that mimic CNS microenvironment [25]. Thus, we speculate that various trophic factors including abovementioned defined factors such as GM-CSF, IL-34, TGF- $\beta$, and cholesterol might be produced from neuroepithelial cells. Second, the supportive capacity of neuroepithelial cells as a feeder gradually decreased as the passage culture of NEL progressed, leading to a decrease in the microglial proliferative capacity. Third, the expression of hexosaminidase subunit beta $(H e x b)$, which is a microglial core gene stably expressed despite the pathological status [26], and colony stimulating factor receptor 1 (Csf1r) was reduced in bNEL-MG compared to pre-banked NEL-MG and there was no difference in Hexb expression between NEL-MG and neonatal microglia. To overcome these limitations, further studies are required to develop an advanced methodology to produce improved NELMG with defined factors, which can induce sustained proliferation, maturation, passage culture, and banking, instead of using neuroepithelial cell feeder.

Regarding the applications of our method, we propose the following as a platform for future use in the neuroscience field: (1) mass production of microglial cells for high-throughput drug screening, and (2) rapid and efficient generation of mutant microglial cells. Compared to neonatal microglia culture, our proposed method enabled us to obtain approximately 30 times more microglial cells when we used a cutoff of 100 culture days. Drug screening for inflammatory modulators targeting neuroinflammation requires significant amounts of microglial cells; however, the currently used in vitro method for primary microglia can barely meet the requirements due to limited cell yields and the number of animals required. Thus, our method contributes to overcoming the main limitation of the currently used technique. In addition, the introduction of a newly reported mutant gene into the NEL allowed the production of mass mutant NEL-MG. Using this methodology, we can rapidly evaluate the functional characteristics of mutant microglia without using a mutant animal generation. Given that the in vivo brain exhibits complex cell-cell interactions supported by contact-dependent signaling from the surrounding cells, mutant NEL-MG might be mixed with a brain organoid to further observe their behavior in a 3D brain environment, based on the fact that NEL-MG is mixed evenly with neurospheroids (Additional file 1: Fig. S4) [12]. In addition, NEL-MG might contribute to the development of a brain organoid with a controllable microglia ratio because the currently used brain organoid does not contain microglial cells, although one study has reported that a brain organoid might innately contain microglial cells [27]. Overall, our proposed method contributes to 3D brain culture and conventional 2D primary microglial culture.

\section{Conclusions}

In conclusion, we expect that our methodology contributes to overcoming the limitations of previous in vitro culture methods for microglia study by leveraging the NEL-MG platform, and increasing the adult-like microglial cell yield. Above all, we believe that our new methodology will reduce dramatically the use of experimental animals and increase the accessibility of microglial research.

(See figure on next page.)

Fig. 5 NEL-MG with a closer fidelity to adult microglia. A Principal component analysis (PCA) was performed to determine the similarity among the BV2 cell line, neonatal microglia (MG), NEL-MG, banked NEL-MG (bNEL-MG), and adult MG. B PCA Euclidean distances to the adult MG were calculated between all pairs of points in each group using PC1, PC2, and PC3 on microglial signature genes. ${ }^{* * *} p<0.00001$. C Clustering heatmap representation of microglia signature gene expression between clusters. The scale represents the median absolute deviation by row. For calculating the distance, a Euclidean distance metric and average linkage clustering were performed using MeV software. D The overall expression changes (z-score normalized) were plotted among groups as mean \pm SD. E Differentially expressed genes between NEL-MG and adult MG. The number of shared genes was 789. $\mathbf{F}$ Gene ontology (GO) analysis of the shared genes (789 genes). G KEGG pathway analysis of the shared genes (789 genes). $\mathbf{H}$ Differentially expressed genes between NEL-MG and neonatal MG. The number of unshared genes in NEL-MG was 2782 (I). GO analysis of the 2782 genes. G KEGG pathway analysis of the 2782 genes. J Upregulated differentially expressed genes between NEL-MG and neonatal MG relative to adult MG were compared regarding the microglial signature genes. The dotted line indicates 1.5 -fold change compared to neonatal MG. ${ }^{*} p<0.05$ 
A.

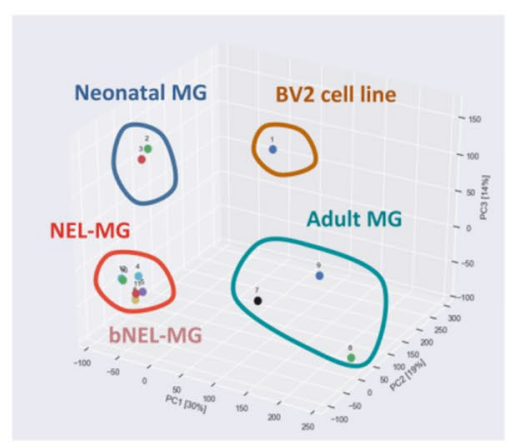

B. Distance to Adult Microglia D

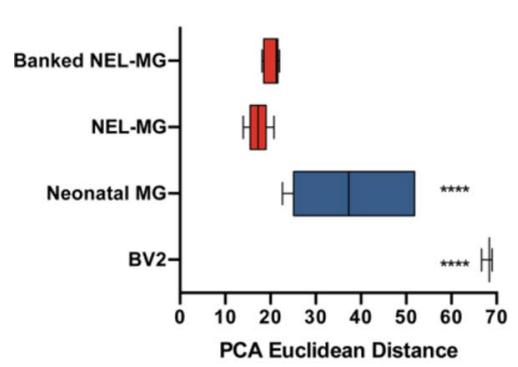

- BV2 cell

- Neonatal MG1

- Neonatal MG2

- NEL-MG1(P0,21d)

- NEL-MG3(P2,40d)

- NEL-MG2(P4,60d)

- Adult MG1

- Adult MG2

- Adult MG3

- Banked NEL-MG1(P1)

- Banked NEL-MG2(P2)

Banked NEL-MG3(P3)

D.

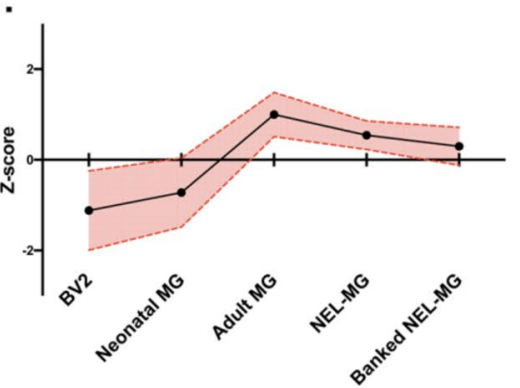

E. NEL-MG

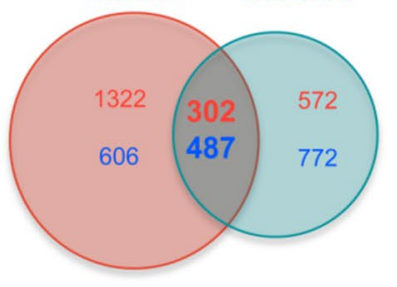

G.

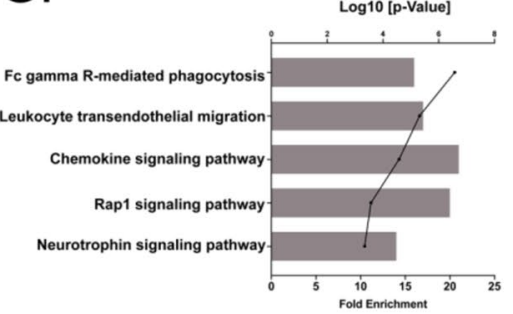

F.
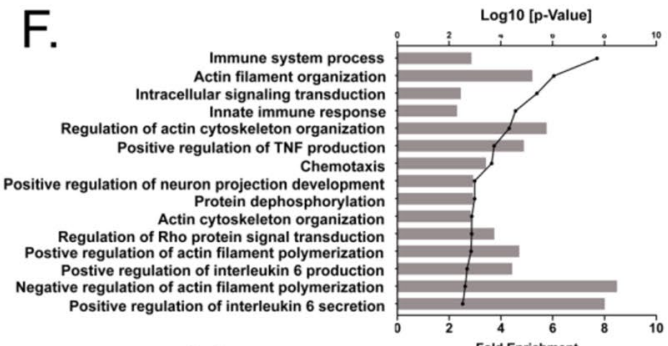

$\mathrm{H}$.
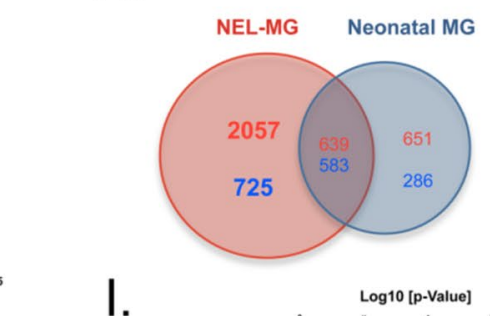

I.

J.
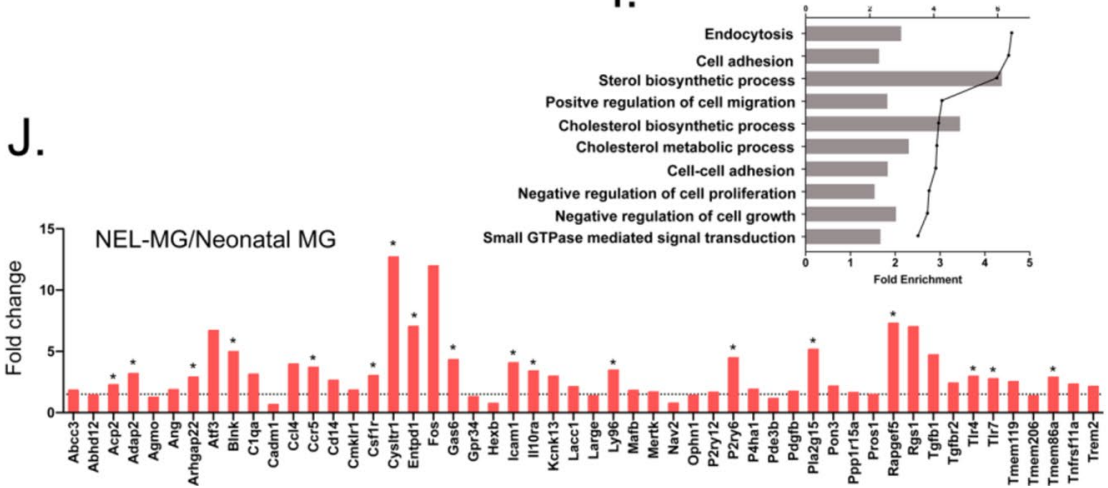

$\begin{array}{ll}\text { BV2 } & \text { NEL MG } \\ \text { Neonatal MG } & \text { Banked NEL MG }\end{array}$ - Adult MG

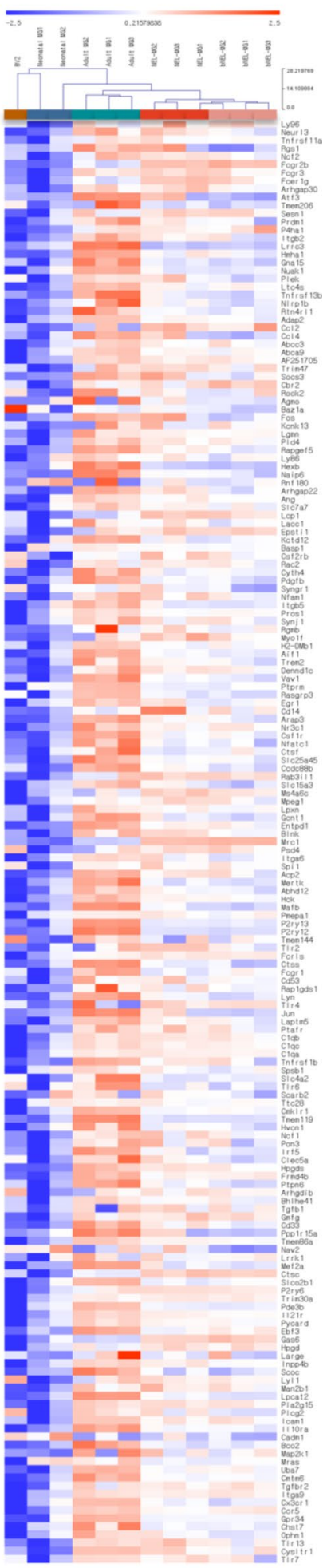

Fig. 5 (See legend on previous page.) 


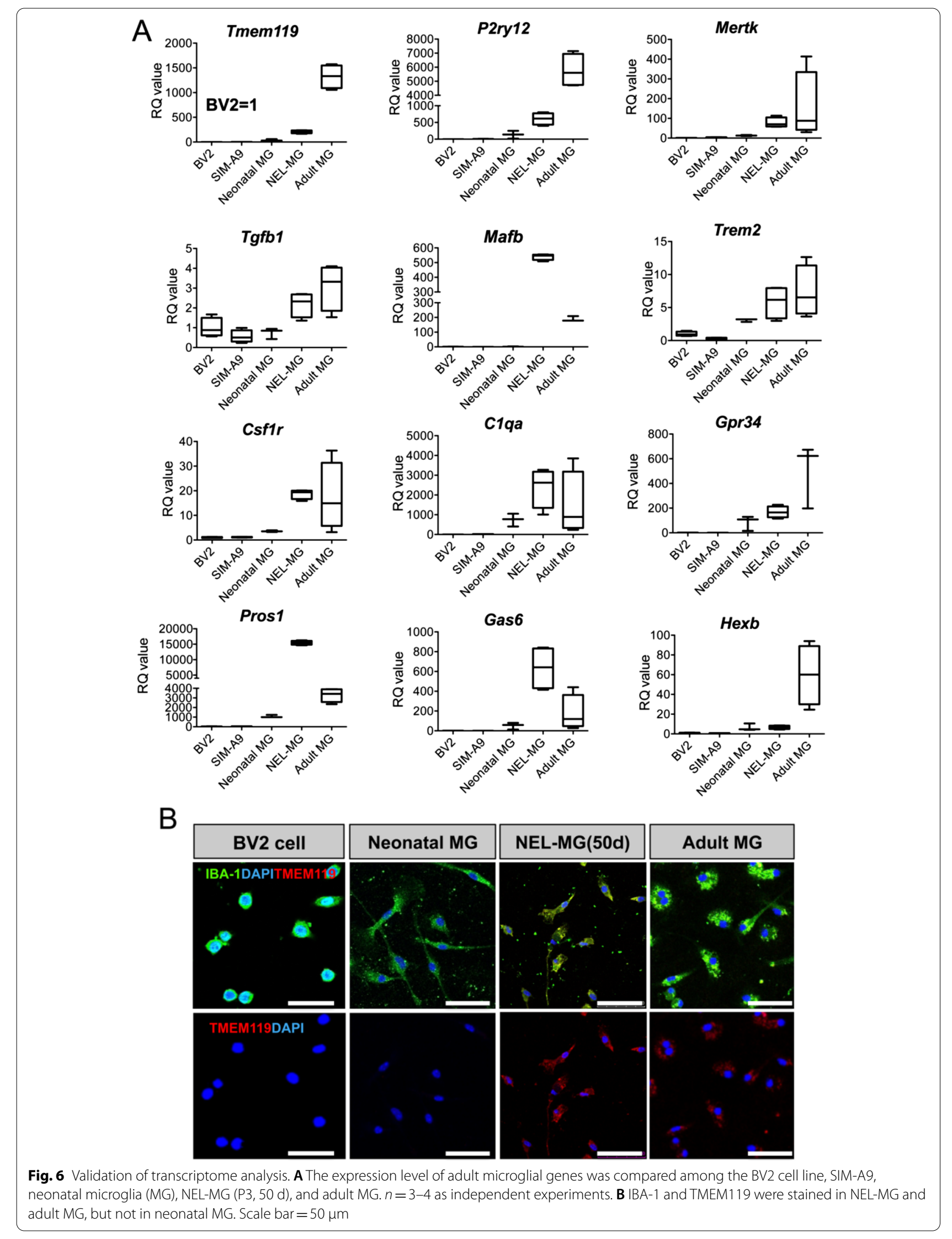




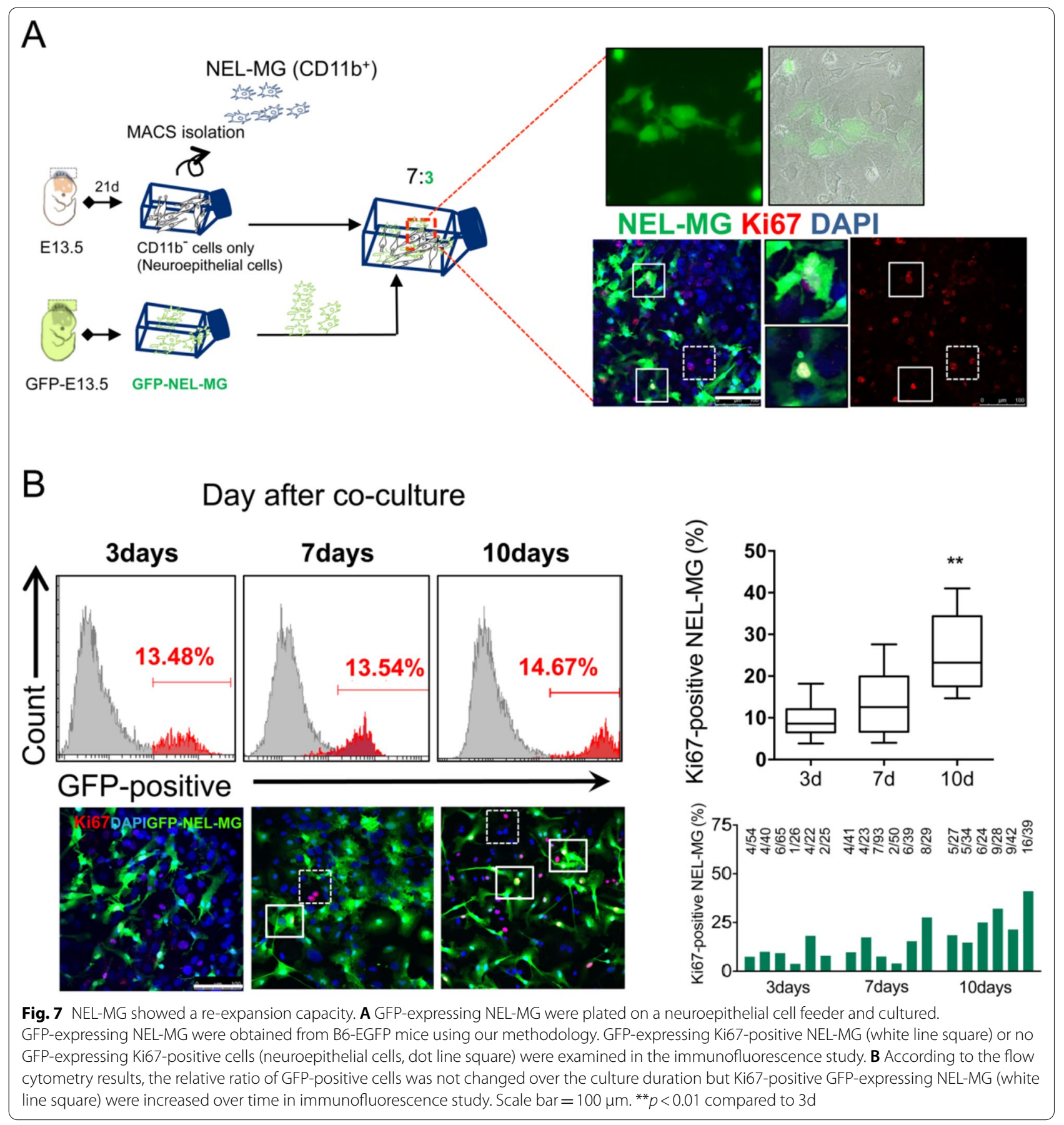




\section{Abbreviations}

CNS: Central nervous system; NEL: Neuroepithelial layer; MG: Microglia; NELMG: CD11b-positive cells isolated from NEL; MACS: Magnetic-activated cell sorting system; LPS: Lipopolysaccharide; iPSC: Induced pluripotent stem cells; PCA: Principal component analysis; GO: Gene Ontology; DEG: Differentially expressed genes.

\section{Supplementary Information}

The online version contains supplementary material available at https://doi. org/10.1186/s12974-021-02351-4.

Additional file 1: Figure S1. Comparison of the cell yields according to the region and embryo stage. Flow cytometry revealed that the dissected head neuroepithelial layer (NEL) from mouse E13.5 could yield a higher ratio of CD1 1b-positive cells than the brain cortex separated from an identical mice group. Immunofluorescence showed that 13.5 NEL have a higher number of IBA-1-positive cells than neuroepithelial layer from mouse E9.5 or E17.5, when cultured for 21 days. Scale bar $=50 \mu \mathrm{m}$. Figure S2. Comparison of phagocytic activity among BV2, neonatal microglia, NEL-MG, and adult microglia. To assess the phagocytic activity, BV2, NELMG, neonatal microglia, and adult microglia at a density of $2 \times 10^{5}$ cells/ $\mathrm{mL}$ were seeded on a $12 \mathrm{~mm}$ coverslip in 24-well cell culture dishes. Each souce of micorglia were treated with $2 \mu \mathrm{L}$ of red fluorescent latex beads ( $2 \mu \mathrm{m}$, Sigma-Aldrich, St. Louis, MO, USA). NEL-MG showed highest phagocytic activity among them. The data are shown as mean \pm standard error of the mean (SEM). Statistical analysis was indicated as a table (below). Figure S3. Mass production of microglial cells via NEC passage culture. Our method produced thirty times the number of microglial cells than that of neonatal microglia when we used a cutoff of 100 days. The data are shown as mean \pm standard error of the mean (SEM). Figure S4. Scatter plot comparison between adult microglia and NEL-MG or neonatal microglia (MG) Diagonal line indicates no significant difference between the two groups (fold change $=1$ ) and the intensity values are normalized to the log2 transformed expression value. Figure S5. Mix with neurospheroid. CellTracker-tagging NEL-MG (red) were mixed with neurospheroids (NS) at different ratios and times. NEL-MG were mixed evenly with NS when we co-mixed them. IBA-1 can label both resident microglia and mixed NEL-MG. Table S1. List of microglial signature genes. Table S2. Primers information.

\section{Acknowledgements}

Not applicable.

\section{Authors' contributions}

YMJ conducted in vitro experiments and wrote the manuscript. RC analyzed transcriptome. KYJ interpreted all data and revised the manuscript. KMS supervised all process, supported experimental conception and design, and approved final submission of manuscript. All authors critically revised the manuscript and confirmed author contribution statement. All authors read and approved the final manuscript.

\section{Funding}

This research was supported by the National Research Foundation of Korea (NRF) grant funded by the Korea government (MIST) (2019M3C7A1032561), by Basic Science Research Program (2020R1F1A1074668), by a grant of the Korea Health Technology R\&D Project through the Korea Health Industry Development Institute (KHIDI), funded by the Ministry of Health \& Welfare, Republic of Korea (HI16C1559). We also appreciate Dr. Yoo J for kindly providing us the B6-EGFP mice.

\section{Availability of data and materials}

All data generated and/or analyzed during the current study are available from the corresponding author on reasonable request.

\section{Declarations}

Ethics approval and consent to participate

All experimental procedures were approved by the Institutional Animal Care and Use Committee (IACUC) of the CHA University (IACUC200116).

\section{Consent for publication}

Not applicable.

\section{Competing interests}

The authors declare that they have no competing interests.

\section{Author details}

${ }^{1}$ Department of Pharmacology, Research Institute for Basic Medical Science, School of Medicine, CHA University, CHA BIO COMPLEX, 335 Pangyo, Bundang-gu, Seongnam-si, Gyeonggi-do 13488, Republic of Korea. ${ }^{2}$ Department of Biochemistry, Research Institute for Basic Medical Science, School of Medicine, CHA University, CHA BIO COMPLEX, 335 Pangyo, Bundang-gu, Seongnam-si, Gyeonggi-do 13488, Republic of Korea.

Received: 23 April 2021 Accepted: 8 December 2021

Published online: 17 December 2021

\section{References}

1. Nayak D, Roth TL, McGavern DB. Microglia development and function. Annu Rev Immunol. 2014;32:367-402.

2. Nimmerjahn A, Kirchhoff F, Helmchen F. Resting microglial cells are highly dynamic surveillants of brain parenchyma in vivo. Science. 2005;308:1314-8.

3. Lenz KM, Nelson LH. Microglia and beyond: innate immune cells as regulators of brain development and behavioral function. Front Immunol. 2018;9:698.

4. Matcovitch-Natan O, Winter DR, Giladi A, Vargas Aguilar S, Spinrad A, Sarrazin S, Ben-Yehuda H, David E, Zelada Gonzalez F, Perrin P, et al. Microglia development follows a stepwise program to regulate brain homeostasis. Science. 2016. https://doi.org/10.1126/science.aad8670.

5. Prinz M, Jung S, Priller J. Microglia biology: one century of evolving concepts. Cell. 2019;179:292-311.

6. Timmerman R, Burm SM, Bajramovic JJ. An overview of in vitro methods to study microglia. Front Cell Neurosci. 2018;12:242.

7. Bennett ML, Bennett FC, Liddelow SA, Ajami B, Zamanian JL, Fernhoff NB, Mulinyawe SB, Bohlen CJ, Adil A, Tucker A, et al. New tools for studying microglia in the mouse and human CNS. Proc Natl Acad Sci U S A. 2016;113:E1738-46.

8. Butovsky O, Jedrychowski MP, Moore CS, Cialic R, Lanser AJ, Gabriely G, Koeglsperger T, Dake B, Wu PM, Doykan CE, et al. Identification of a unique TGF-beta-dependent molecular and functional signature in microglia. Nat Neurosci. 2014;17:131-43.

9. Butovsky O, Siddiqui S, Gabriely G, Lanser AJ, Dake B, Murugaiyan G, Doykan CE, Wu PM, Gali RR, lyer LK, et al. Modulating inflammatory monocytes with a unique microRNA gene signature ameliorates murine ALS. J Clin Invest. 2012;122:3063-87.

10. Guo L, Rezvanian A, Kukreja L, Hoveydai R, Bigio EH, Mesulam MM, El Khoury J, Geula C. Postmortem adult human microglia proliferate in culture to high passage and maintain their response to amyloid-beta. J Alzheimers Dis. 2016:54:1157-67.

11. Muffat J, Li Y, Yuan B, Mitalipova M, Omer A, Corcoran S, Bakiasi G, Tsai LH, Aubourg P, Ransohoff RM, Jaenisch R. Efficient derivation of microglia-like cells from human pluripotent stem cells. Nat Med. 2016;22:1358-67.

12. Abud EM, Ramirez RN, Martinez ES, Healy LM, Nguyen CHH, Newman SA, Yeromin AV, Scarfone VM, Marsh SE, Fimbres C, et al. iPSC-derived human microglia-like cells to study neurological diseases. Neuron. 2017;94:27893.e9.

13. Hoeffel G, Chen J, Lavin Y, Low D, Almeida FF, See P, Beaudin AE, Lum J, Low I, Forsberg EC, et al. C-Myb(+) erythro-myeloid progenitor-derived fetal monocytes give rise to adult tissue-resident macrophages. Immunity. 2015;42:665-78. 
14. Gosselin D, Skola D, Coufal NG, Holtman IR, Schlachetzki JCM, Sajti E, Jaeger BN, O'Connor C, Fitzpatrick C, Pasillas MP, et al. An environmentdependent transcriptional network specifies human microglia identity. Science. 2017. https://doi.org/10.1126/science.aal3222.

15. You MJ, Bang M, Park HS, Yang B, Jang KB, Yoo J, Hwang DY, Kim M, Kim B, Lee SH, Kwon MS. Human umbilical cord-derived mesenchymal stem cells alleviate schizophrenia-relevant behaviors in amphetaminesensitized mice by inhibiting neuroinflammation. Transl Psychiatry. 2020:10:123.

16. Najafi AR, Crapser J, Jiang S, Ng W, Mortazavi A, West BL, Green KN. A limited capacity for microglial repopulation in the adult brain. Glia. 2018;66:2385-96.

17. Bohlen CJ, Bennett FC, Tucker AF, Collins HY, Mulinyawe SB, Barres BA. Diverse requirements for microglial survival, specification, and function revealed by defined-medium cultures. Neuron. 2017;94:759-73.e8.

18. Tanaka J, Maeda N. Microglial ramification requires nondiffusible factors derived from astrocytes. Exp Neurol. 1996;137:367-75.

19. Elmore MR, Najafi AR, Koike MA, Dagher NN, Spangenberg EE, Rice RA, Kitazawa M, Matusow B, Nguyen H, West BL, Green KN. Colony-stimulating factor 1 receptor signaling is necessary for microg lia viability, unmasking a microglia progenitor cell in the adult brain. Neuron. 2014;82:380-97.

20. Deczkowska A, Amit I, Schwartz M. Microglial immune checkpoint mechanisms. Nat Neurosci. 2018;21:779-86.

21. Pfrieger FW, Ungerer N. Cholesterol metabolism in neurons and astrocytes. Prog Lipid Res. 2011;50:357-71.

22. Rapp A, Gmeiner B, Huttinger M. Implication of apoE isoforms in cholesterol metabolism by primary rat hippocampal neurons and astrocytes. Biochimie. 2006;88:473-83.

23. Lee CY, Tse W, Smith JD, Landreth GE. Apolipoprotein E promotes betaamyloid trafficking and degradation by modulating microglial cholesterol levels. J Biol Chem. 2012;287:2032-44.

24. Loving BA, Bruce KD. Lipid and lipoprotein metabolism in microglia. Front Physiol. 2020;11:393.

25. Gotz M, Huttner WB. The cell biology of neurogenesis. Nat Rev Mol Cell Biol. 2005:6:777-88.

26. Masuda T, Amann L, Sankowski R, Staszewski O, Lenz M, Snaidero N, Costa Jordao MJ, Bottcher C, Kierdorf K, et al. Novel Hexb-based tools for studying microglia in the CNS. Nat Immunol. 2020;21:802-15.

27. Ormel PR, de Vieira Sa R, van Bodegraven EJ, Karst H, Harschnitz O, Sneeboer MAM, Johansen LE, van Dijk RE, Scheefhals N, van Berdenis Berlekom A, et al. Microglia innately develop within cerebral organoids. Nat Commun. 2018:9:4167.

\section{Publisher's Note}

Springer Nature remains neutral with regard to jurisdictional claims in published maps and institutional affiliations.

Ready to submit your research? Choose BMC and benefit from:

- fast, convenient online submission

- thorough peer review by experienced researchers in your field

- rapid publication on acceptance

- support for research data, including large and complex data types

- gold Open Access which fosters wider collaboration and increased citations

- maximum visibility for your research: over $100 \mathrm{M}$ website views per year

At BMC, research is always in progress.

Learn more biomedcentral.com/submissions 